Revista de Filología Románica

ISSN: 0212-999X

http://dx.doi.org/10.5209/RFRM.63507

\title{
mecum, cum mecum, conmigo y con mí: saber lingüístico y morfología de prestigio
}

\author{
Frank Jodl ${ }^{1}$
}

Recibido: 22 de marzo de 2018 / Aceptado: 26 de septiembre de 2018

Resumen. Interpretando al Codex emilianense Em 31 y el glosario latino-latino copiado en él (scriptorium de San Millán de la Cogolla, siglo X o XI), Claudio García Turza (2004, 112s.) destaca, entre otras, la voz cum me que la glosa confronta con mecum. Dado que en el castellano moderno no se ha impuesto ninguna de las dos variantes, trataré de remontar los orígenes de la forma "vencedora" pleonástica cum mecum, o sea, conmigo basándome en el comentario metalingüístico medieval representado por la glosa. El objetivo del presente artículo es demostrar la idoneidad del concepto de la morfología de prestigio (Jodl 2006, 12-16 y Jodl 2018, 275-280) para explicar el fenómeno en cuestión y para completar los modelos del cambio lingüístico en general.

Palabras clave: sociolingüística histórica; cambio lingüístico; morfología de prestigio; saber lingüístico; conciencia lingüística

\section{mecum, cum mecum, conmigo and con mí: linguistic knowledge and prestige- morphology}

\begin{abstract}
Interpreting the Codex emilianense Em 31 and the Latin-Latin glossary included in it (scriptorium San Millán de la Cogolla, 10th or 11th century), Claudio García Turza (2004, 112s.) emphasizes, among other things, the entry cum me that the gloss compares to mecum. Due to the fact that neither of the two forms has survived in modern Spanish, I shall attempt to trace back the origins of the imposing (pleonastic) form cum mecum, i.e. conmigo, basing my assumptions on the metalinguistic comment represented by the gloss. My goal will be to illustrate that the phenomenon in question can be explained by the prestige morphology concept (Jodl 2006, 12-16 and Jodl 2018, 275-280), which can furthermore be used to complete the current linguistic change theories in general.
\end{abstract}

Keywords: historical sociolinguistics; linguistic change; prestige morphology; linguistic knowledge; linguistic awareness

Sumario: 1. Introducción. 2. Posibles respuestas a las preguntas a.) - f.). 3. ¿Qué papel desempeñan en este caso planteamientos como la morfología "junk", la "exaptación", la "capitalización" y "la refuncionalización"? 4. Propuesta de un planteamiento ulterior: la morfología de prestigio y sus implicaciones con el saber y el comportamiento lingüísticos y la koineización. 5. Conclusiones

Cómo citar: Jodl, F. (2019). mecum, cum mecum, conmigo y con mí: saber lingüístico y morfología de prestigio, en Revista de Filología Románica 36, 89-111.

\footnotetext{
TH Köln

frank.jod1@th-koeln.de
} 


\section{Introducción ${ }^{2}$}

Interpretando al Codex emilianense Em 31 y el glosario latino-latino copiado en él (scriptorium de San Millán de la Cogolla, siglo X o XI), Claudio García Turza (2004: 112-113) destaca, entre otras, la voz cum me:

(1) cum me: sic dicebant, et cum te; quod nunc elegantjus dicimus mecum, tecum $(9 \mathrm{r} 1,19)$

García Turza (ibid.) nos da, al respecto, el siguiente comentario:

Recuérdese que además de los descendientes iberorrománicos de los lexemas considerados inseparables MECUM mego, TECUM tego, y de los que produjo la lexicalización de los sintagmas pleonásticos CUMMECUM co(n)migo, CUMTECUM contigo, son también interesantes, los herederos de la construcción analítica, CUM ME con mí, CUM TE con ti, que conservan todavía hoy no poca vitalidad, por ejemplo, en Aragón, La Rioja, Soria, etc.

Si tenemos en cuenta que esta glosa ya figura en una obra glosemática anterior (¿carolingia?), concretamente en el Liber Glossarum (LGl, 78r, cf. García Turza, ibd., p. 97), e involucramos las soluciones morfológicas de las demás variedades romances (italiano: con me; variedad castellana de la provincia de Soria: con mí, etc., véase también abajo, 2.2) podremos concluir lo siguiente: $\mathrm{La}$ evolución ${ }^{3}$ pan-románica incluyendo en gran parte el iberorromance se decantó, respecto a este caso, por el tipo morfológico CUM ME. Sin embargo, en la variedad estándar del castellano se impuso el tipo pleonástico CUM MECUM: El elemento enclítico latino -CUM, que según las leyes fonéticas "tuvo" que convertirse en "-go", dejó de ser semánticamente transparente, se fosilizó y fue re-analizado como "perteneciente al pronombre ME-/ $m i-;$ Rini $1990 \mathrm{a}+\mathrm{b})$. Si seguimos este principio y si las apariencias no nos engañan, resulta relativamente fácil describir la gestación de la innovación "vencedora". Sin embargo, nuestra glosa, tematizada por García Turza, nos brinda varias pistas para hacer ulteriores preguntas:

a.) ¿Cómo interpretar las formas cultas CUM ME y MECUM? Para un esbozo acerca del comportamiento lingüístico véase párrafo 2.1, abajo

b.) ¿Quién utilizaba "antes" CUM ME ("sic dicebant") y qué quiere decir "antes"? Véase párrafo 2.2, abajo

c.) ¿Quién utiliza "ahora" MECUM ("nunc [...] dicimus) y qué quiere decir "ahora"? Véase párrafo 2.3, abajo

d.) ¿Cómo podemos interpretar "elegantjus" ("nunc elegantjus dicimus")? Véase párrafo 2.4 , abajo

e.) ¿Cómo se puede explicar que no se impusieran ni la forma previsible CUM ME, ni el -como parece- igualmente previsible MECUM? Véase párrafo 2.5, abajo

2 Este artículo representa la versión ampliada de la charla que hice en ocasión del Hispanistentag 2015, o sea, del XX Congreso de la Asociación Alemana de Hispanistas 2015 (que se celebró del 18 al 22 de marzo). Quisiera brindarles mis más sinceras gracias a Michel Leiberich y Xavier Roca por haberme asistido a la hora de la relectura del texto. Cualquier error que aún contenga corresponde -obviamente- a mi responsabilidad. Quisiera agradecer también a Rosa M. Espinosa Elorza y Emiliana Ramos Remedios por las valiosas sugerencias que me han hecho.

3 Quiere decir el uso que hicieron los locutores, cf. Kabatek (2005). 
f.) ¿Qué papel desempeñan en este caso planteamientos como la morfología “junk" y la "exaptación” (Lass 1990 y 1997, Espinosa Elorza 2008), la "capitalización" (Pountain 2000), la refuncionalización (Smith 2011) y factores como el saber lingüístico y la koineización (Tuten 2003)? Véanse párrafos 2.5 y 3 ., abajo

Planteando estas cuestiones, intentaré acercarme a un concepto ulterior que podría contribuir a la aclaración de nuestro problema y que llamaré morfología de prestigio (Jodl 2006, 12-16), véase párrafo 4., abajo.

\section{Posibles respuestas a las preguntas a.) $-\mathbf{f}$.)}

\section{1 ¿Cómo interpretar las formas cultas CUM ME y MECUM? Esbozo acerca del comportamiento lingüístico ${ }^{4}$}

Antes de poder afrontar las preguntas b.) - g.) tendremos que ocuparnos de un tema muy genérico (véase arriba pregunta a.): ¿Cómo podemos interpretar el hecho de que en el comentario metalingüístico del siglo $\mathrm{X}$, refiriéndose al lenguaje hablado de la época, aparezcan formas cultas o incluso del latín clásico? Es en este contexto que vuelvo a la pregunta que ya hice en Jodl (2010: especialmente 136, 142, 153-154):

¿Es posible que en ciertos ámbitos sociales, como el eclesiástico, los individuos aspirasen a practicar, incluso en su vida de todos los días, una latinidad "más auténtica" / "más clásica"? Es interesante la pregunta porque recomendaciones lingüísticas concebidas por movimientos culturales como la "renaissance carolingienne" no se respetarían exclusivamente en el ámbito de la escripturalidad, sino también en el de la oralidad.

Para facilitar las reflexiones siguientes establezco aquí los siguientes escenarios de comportamiento lingüístico que voy a tratar más detalladamente.

Partiendo de la presuposición sociológica de que la elección de High / Low Varieties $^{6}$ desempeña un papel crucial respeto del comportamiento lingüístico, sigo en un principio las aclaraciones de Elias (1997/1939: 237-244) quien se basa en De Callières (1693/1972). En De Callières encontramos informaciones metalingüísticas, concretamente informaciones acerca de las posturas que asumían los hablantes a propósito de las distintas variedades ${ }^{7}$ lingüísticas repartidas en la sociedad. Por lo

4 Más concretamente: El comportamiento lingüístico de los grupos sociales involucrados en el proceso que estamos describiendo, a la hora de escoger variedades lingüísticas, cf. Labov (1963 y 2010).

5 Si es aceptable esta denominación se discute en Depreux (2002/3).

6 Utilizando los términos "High" y "Low variety" me refiero a la concepción acuñada por Ferguson (1959), cf. también Koch (1997: 222) que defiende este concepto original (destacando las desventajas de posteriores ensanches, id. ibd. 220-221).

$7 \quad$ El presente artículo trata de una problemática medieval. No puedo retomar aquí la discusión desencadena por Roger Wright (1982), cf. también Ramos Remedios (2000: 26-33) y Richter (1983), que trata la pregunta: ¿A partir de cuándo el latín y el romance ya no pueden ser considerados como una misma lengua histórica? Por lo que toca al tema tratado en el artículo presente, parto del principio de que en la Península Ibérica habría -en comparación a la situación vigente en Galia- un retraso en cuanto a la escisión de latín y romance. Sin embargo, no me parece aceptable (cf. Wolf 1991: 53, 60-62, 91s./trad. esp. Wolf 1996) la visión de Wright (1982: p. ej. 204) según la cual durante nuestra época (aprox. año 1000) el latín y el romance seguirían siendo la misma lengua. Cf. también el resumen en Jodl (2016a: 94-96) y Wolf (1997). Aunque Wright tenga razón afirmando 
que concierne a la actitud que asumía la nobleza frente a la burguesía, cf. De Callières (1693/1972: 46):

Vous sçavez que les Bourgeois parlent tout autrement que nous.

Pero volvamos al trabajo de Elias del que podemos sacar conceptos cruciales que resumiría como sigue:

I.) Elias nos presenta ejemplos metalingüísticos auténticos ${ }^{8}$ que muestran cuál era el auto-concepto de los hablantes pertenecientes a la nobleza (en lo referente al prestigio de su lenguaje). De De Callières (1693/1972: 23, letra negrita F.J.) cita lo que nos hace saber una noble hablando a un joven burgués: ${ }^{9}$

Il est fort possible qu'il y ait quantité d'honnêtes gens qui ne connoissent pas assez la délicatesse de nôtre Langue, ... cette délicatesse qui n'est connue que d'un petit nombre de gens qui parlent bien, qui fait qu'ils ne disent point qu'un homme est deffunct, pour dire qu'il est mort.

II.) La instancia que decide qué es "hablar bien" y qué es "hablar mal" es el uso practicado por la nobleza, cf. Elias (1997/1939: 241).

III.) "Nuevos"10 usos (provenientes de ámbitos sociales inferiores) no pueden establecerse sin la aceptación de la nobleza, es decir: Si la nobleza no pasa a servirse también de los nuevos usos, éstos no se impondrán, cf. Elias (1997/1939: 239) y Coseriu (1974/1958: 68). ${ }^{11}$

IV.) Elias trata exclusivamente el hablar correctamente ("parler bien", p. ej. id., ibd. p. 241), parece dar por descontado que "el escribir" tendrá (automáticamente) la misma calidad si efectuado por un individuo que habla "bien". Esto, por principio, coincide con Jodl (2010) donde hago hincapié en que hay locutores cuyo uso de la lengua casi no manifiesta diferenciación entre "uso escrito" y "uso hablado".

Si partimos del concepto de que el comportamiento lingüístico descrito por Elias es un fenómeno universal (lo que insinúan los ejemplos de otras épocas que presentaré abajo) y si por eso transferimos las indicaciones que nos dio Elias a la situación concreta que nos interesa aquí, o sea, a los locutores involucrados en el continuum latín - romance llegamos al

que tanto el latín clásico como el latín vulgar/romance forman parte de una misma lengua HISTÓRICA, el latín, puede equivocarse en cuanto a la situación socio-cultural concreta: El nacimiento de "nuevas" lenguas (a partir de variedades de lenguas históricas) se debe a una construcción social y son los hablantes y procesos históricos que contribuyen a la formación de la consciencia, según el principio: La variedad X de la lengua $\mathrm{Z}$ deja de ser variedad, o sea, se ha convertido en "nueva lengua", cf. Jodl (2002/3: 169-171) por un resumen acerca del desarrollo del concepto de "lengua histórica" y en general Ramos Remedios (2016).

8 Respecto al concepto de "autenticidad” asumo aquí la postura de Depreux (2002/3: 731), cf. también la citación abajo, presente párrafo, resumen.

9 Quien acaba de intentar defender su mal uso de la lengua, cf. Elias (1939/1997: 241).

10 En cuanto al carácter de la innovación lingüística cf. Kabatek (2005) y Coseriu (1974/1958).

11 Así que la actual lengua nacional se basa muy ampliamente en los usos de la nobleza, cf. Elias (1939/1997: $237+240)$, lo que no impide que este lenguaje "noble" haya sido integrado por formas provenientes de otros ámbitos sociales. En cuanto a procesos de koineización cf. Tuten (2003). 


\section{Escenario a.):}

Habría, en la edad media, locutores cultos, según Depreux (2002/3: 722) "des lettrés", que -en el sentido de Jodl (2010: espec. 136, 142, 153-154)- se atenían incluso en el marco de la comunicación oral (cum grano salis) a la norma clásica del latín, lo que podría insinuar

a1.) El "Proemio" del "Novellino": 12

E acciò che li nobili e gentili sono nel parlare e ne l'opere quasi com'uno specchio appo i minori, acciò che il loro parlare è più gradito, però ch'esce di più dilicato stormento, facciamo qui memoria d'alquanti fiori di parlare [...].

Traducción al español F.J.:

$Y$ visto que los nobles y gentiles son -en cuanto a su manera de hablar y sus obras-casi como un espejo para la gente de condiciones más humildes, lo que se debe al hecho de que la manera de hablar de los nobles es más fina porque sus palabras salen de un instrumento más delicado, retenemos aquí algunas flores de su arte de hablar.

Además tenemos como fuentes:

a2.) Los testimonios del escenario b.) de los que no se desprende tan sólo que existía la capacidad y postura para conmutar ${ }^{13}$-en función de la situación comunicativa y el propósito- entre por lo menos dos variedades distintas de una lengua histórica, ${ }^{14}$ sino también la capacidad y el auto-concepto de (aspirar a) practicar la norma clásica en todas las situaciones de la vida, cf. resumen del presente párrafo, abajo.

Teniendo en cuenta que en el trabajo presente tratamos una problemática medieval que coincide con la tratada en Jodl (2010), querría precisar la tesis defendida ibd. ofreciendo un tipo suplementario:

\section{Escenario b.)}

Locutores que -adaptándose a situaciones específicas y a los objetivos comunicativos- eran capaces de expresarse en distintas variedades del latín, o sea, variedades con más o menos prestigio.

Esta flexibilidad lingüística se puede precisar como sigue:

Con más prestigio $\rightarrow$ variedades que están cerca de la norma clásica del latín

Con menos prestigio $\rightarrow$ variedades del romance

\section{Ejemplo 1:}

La "Vita Eligii"'5 nos brinda un excelente ejemplo de la flexibilidad lingüística que cundía en el ámbito social y durante la época que nos interesan, cf. Depreux (2003: 745):

12 En lo siguiente trataré de mostrar que lo que se afirma aquí acerca del medioevo tardío se puede transferir a la situación del medioevo temprano.

13 Así que las fuentes primarias medievales afirman que el desvío de la norma clásica era intencional / deliberado.

14 Véase arriba, nota 7.

15 Cf. la "Vita Eligii" en: Prol. MGH SS rer. merov. IV, 634-761, especialmente p. 664. 
'L'écriture d'un texte destiné au peuple supposait en effet qu'on s'adaptât à ses usages linguistiques, comme le prouvent les propos de l'auteur de la Vie de saint Éloi qui, dans la seconde moitié du VIIIe siècle, déclare adapter son style à un auditoire illettré $[\ldots] . "{ }^{\prime 16}$

\section{Ejemplo 2:}

Una actitud análoga se desprende de la "Vita Beatissimi Richarii Presbyteri"17, cf. Depreux (2003: 746): 18

[...] les lettrés de l'époque carolingienne avait d'autres exigences. Cela est très nettement illustré par la rédaction d'une nouvelle Vie de Saint Riquier par Alcuin, en 801 : les moines de Saint-Riquier utilisaient un texte mérovingien pour l'édification du peuple, mais eux-mêmes souhaitaient disposer d'une version à la grammaire plus correcte pour les usages monastiques.

\section{Ejemplo 3:}

Más difícil es el caso de Gregorio de Tours. Sus obras siempre han servido de ejemplo de un latín ya muy corrompido (cf. Plassmann 2006: 117-118) y es Gregorio mismo que nos inspira esta manera de ver las cosas pidiendo disculpa en el proemio por su latín inculto y rústico. Pero hoy en día sabemos más acerca del concepto lingüístico que se nos presenta en sus textos. La idea ${ }^{19}$ de que el latín de Gregorio fuera de pobre calidad, porque su competencia lingüística no fue suficiente para alcanzar el nivel clásico, es rechazada ${ }^{20}$ por la mayoría de los historiadores: Quiere decir que según las opiniones más modernas ${ }^{21}$ Gregorio adaptó su registro deliberadamente a las competencias de sus lectores. Él mismo, con toda probabilidad, dominó otros registros del latín, también los más elevados. Si percibimos de esta manera la estrategia lingüística perseguida por Gregorio, el ejemplo de su caso coincide con los demás ejemplos.

16 Cf. a propósito de la "Vita Eligii”" Heen (1991, 151).

17 Cf. Alcuinus "Vita Beatissimi Richarii Presbyteri”, en: Migne Jacques-Paul, Patrologia latina 101, 681-694.

18 La citación completa sería: «L'écriture d'un texte destiné au peuple supposait en effet qu'on s'adaptât à ses usages linguistiques, comme le prouvent les propos de l'auteur de la Vie de saint Éloi qui, dans la seconde moitié du VIIIe siècle, déclare adapter son style à un auditoire illettré [...]. En revanche, les lettrés de l'époque carolingienne avait d'autres exigences. Cela est très nettement illustré par la rédaction d'une nouvelle Vie de Saint Riquier par Alcuin, en 801 : les moines de Saint-Riquier utilisaient un texte mérovingien pour l'édification du peuple, mais eux-mêmes souhaitaient disposer d'une version à la grammaire plus correcte pour les usages monastiques." (Depreux 2002/3: 746).

19 Cf. Bonnet (1890: 76s.). La idea de que el latín producido por Gregorio fuera de pobre calidad se desprendía también de la manera de la que Krusch/Levinson (1937, eds.) representaron los textos de Gregorio de Tours.

20 Cf. contra Krusch, o sea Krusch/Levinson (1937, eds.), los trabajos Bourgain/Heinzelmann (1997: 294f.), Goffart (1987/1989a: 257), Goffart (1989b: $177+179$ ss. + 197-203). Importantes son, sobre todo, las recensiones a la edición Krusch, Bruno/Levison, Wilhelm (1937, eds.): Scriptores rerum Merovingicarum 1,1: Gregorii Turonensis Opera. Teil 1: Libri historiarum X. Hannover 1937 (Monumenta Germaniae Historica), redactadas por Buchner (1942, 1951, 1955, en las que se basa Goffart 1987/1989a: 257). Cf. sobre todo Buchner (1942: 411).

21 Cf. el resumen en Müller (2001: 73-78) donde la argumentación se basa en Fuhrmann (1994: 345+346) y además en Berschin (1986: 299-302), Buchner ( ${ }^{9} 2000$, introducción pág. XL) y Beumann (1964: espec. 81f.). Con más reserva se expresa Plassmann (2006: 119f.). Es interesante que también Bonnet (1890), véase nota arriba, quien normalmente hace hincapié en que Gregorio carecía de cultura y competencias lingüísticas no descuida subrayar que Gregorio, por otro lado, era capaz de elegir niveles de estilo más altos, cf. Bonnet (1890: 744ss.). 
Retengamos como resumen: El hecho de que el escenario b.) nos enseñe que también en el ámbito eclesiástico se hablaba romance ${ }^{22}$ no quiere decir que faltase el latín clásico, cf. Bork $^{23}(2006,1583$, letra negrita F.J.):

Das Lateinische ist im ganzen katholischen Europa des Mittelalters [...] omnipräsent, zunächst als einzige, dann als dominante Schriftsprache und als Sprache der Alphabetisierung, weiterhin als gesprochene [sic] Sprache der Kirche.

[Traducción F.J.: El latín es omnipresente en la Europa católica de la Edad media, primero como la única lengua escrita que en siglos posteriores se convirtió en la lengua dominante y de alfabetización. Además, el latín mantuvo su función como única lengua hablada [sic!] de la iglesia.]

En otras palabras: En el ámbito eclesiástico se utilizaban como lenguas habladas tanto el latín clásico como el romance, que -como dijimos (véase la nota 7 , arriba) - en cuanto a la Península Ibérica de los siglos en cuestión pueden ser considerados como variedades de la misma lengua histórica. Consecuentemente (cf. Jodl 2010) puede haber habido grupos sociales en los que los individuos disponían -cum grano salis- de tan sólo una variedad lingüística, cerquísima de o idéntica a la variedad "Distanzsprache", ${ }^{24}$ en el caso concreto: el latín clásico, véase el comportamiento lingüístico de las élites. ${ }^{25}$

Eso dependería del auto-concepto de los hablantes y de la "presión" correctiva que emanaba de la "renaissance / réforme carolingienne", cf. Depreux (2003, 732s., letra negrita F.J.):

À propos du Haut Moyen Âge, on a écrit que le maître mot n'est pas celui de renaissance, mais celui de réforme [...] (qui trouve notamment son expression dans la réforme monastique déjà sensible sous Charlemagne et généralisée sous Louis le Pieux [...]). À vrai dire, l'idée essentielle est celle de correction [...]. La manière dont Notker le Bègue relate ${ }^{26}$ l'anecdote de l'inspection de la classe de Clément est particulièrement significative. Charlemagne félicite les bons élèves (d'origine modeste [...]) de leur travail et les incite à tendre vers la perfection : Nunc ergo ad perfectum attingere studete ${ }^{27}[. .$.$] . C'est à cette condition que le souverain est$ censé leur attribuer quelque épiscopat ou abbatiat prestigieux. On retiendra ici que pour le moine de Saint Gall, comme pour nombre de ses contemporains, l'étude était un préalable, un chemin conduisant à la perfection (de même que pour Hincmar de Reims l' «école du palais» était avant tout une école de vie ${ }^{28}$ ).

22 En MGH, Abt. I: Scriptores 2 [Scriptores rerum Sangallensium. Annales, chronica et historiae aevi Carolini], 121s. Ekkehard IV. hace constar un episodio según el que el abad Ekkehard I. de San Galo (fallecido en 973) exhortó «teutonice et romanice» a dos monjes, cf. también Kramer (1998, 85-86).

23 Cf. también Pierno (2006: 2071) y generalmente Jodl (2010: 140).

24 Cf. Koch/Oesterreicher (1985) y Koch/Oesterreicher (2011/1990).

25 Cf. acerca del comportamiento lingüístico de las élites Elias (1997: 195 y 237-44), Reutner (2009: 195ss.) y el resumen en Reutner/Schwarze (2011: 223). Cf. también Bourdieu (1984/2002 : 123) y Delhees (1994: 224).

26 Por lo que concierne a la fiabilidad de Notker cf. Depreux (2002/3, 731): “On sait que Notker a inventé beaucoup de détails et d'anecdotes ; ce qui est important, ce n'est pas de savoir si ce prêtre exista réellement, mais d'observer que le moine de Saint-Gall tenait cela pour tout à fait vraisemblable."

27 Cf. Notker Balbulus Gesta Karoli Magni [n. 3], p. 4 (I, c. 3).

28 Capitularia regum Francorum, t. 2, éd. Alfred Boretius et Victor Krause, Hannover, Monumenta Germaniae Historica, 1890, no 297, p. 436 (Epistola synodi Carisiacensis ad Hludowicum regem Germaniae directa, c. 
Así nos sea permitido presuponer que las admoniciones emanando de la "renaissance carolingienne" surtieron su efecto ${ }^{29}$ proporcionando a ciertos grupos de individuos cultos la voluntad y capacidad de aspirar a reproducir el estándar clásico del latín también en el marco de la comunicación de todos los días.

Un ejemplo concreto sería el que nos brinda Bourdieu mencionando que sus profesores aun utilizaban el "subjonctif de l'imparfait" (por lo menos el de la tercera persona del singular) conscientemente para dar más valor a su lenguaje, cf. Bourdieu (1984/2002: 123, letra negrita F.J.):

Par exemple, sur le marché scolaire l'imparfait du subjonctif recevait une grande valeur du temps de mes professeurs qui identifiaient leur identité professorale au fait de l'employer - au moins à la troisième personne du singulier - ce qui, aujourd'hui, ferait sourire et n'est plus possible devant un public d'étudiants, sauf à faire un signe métalinguistique pour marquer qu'on le fait mais qu'on pourrait ne pas le faire. ${ }^{30}$

Que algunos científicos sigan utilizando el "subjonctif de l'imparfait" en la actualidad, lo vemos confirmado en el texto de Depreux (al que -hasta ahora- nos hemos referido por motivos metalingüísticos y no como fuente primaria de fenómenos lingüísticos). Depreux no tan sólo utiliza "le subjonctif de l'imparfait", sino además ensancha su uso, quiere decir: En los casos que vamos a citar, Depreux utiliza incluso el plural de la tercera persona, cf. Depreux (2002/2003: 723, letra negrita F.J.):

C'était une tradition ancienne que les jeunes gens de l'aristocratie vinssent au palais pour y être nourris.

y Depreux (2002/2003: 729, letra negrita F.J.):

Bien que les réminiscences virgiliennes fussent de règle, certains auteurs n'hésitaient pas à s'inspirer d'autres poètes, tel Ermold le Noir, qui chercha à gagner la clémence de Louis le Pieux en louant son gouvernement ; [...]

Todos estos ejemplos demuestran que existen condiciones que, según Elias, pueden explicar por qué ciertos individuos poseen tan sólo una variedad: la clásica, cf. también Jodl (2010).

Ahora bien, para explicar la veste clásica de nuestra glosa se podría esgrimir que el autor medieval -por tratarse de un comentario escrito (aunque se refería a formas populares) - se haya adaptado, tan simplemente, con cierto automatismo al uso de la escrituralidad en una única situación de redacción: Pero si se tratase de verdad de

12): "Et ideo domus regis scola dicitur, id est disciplina ; quia non tantum scolastici, id est disciplinati et bene correcti, sunt, sicut alii, sed potius ipsa scola, quae interpretatur disciplina, id est correctio, dicitur, quae alios habitu, incessu, verbo et actu atque totius bonitatis continentia corrigat."

29 Cf. Depreux (2002/3: 746), véanse las citaciones arriba.

30 En este contexto, Bourdieu (ibd.) utiliza el término "hypercorrection (controlée)". Para evitar confusiones destaco aquí que este uso del término no corresponde al uso normal de "hypercorrection" como término específicamente lingüístico (que se refiere también a la producción de errores como infracción del sistema), véase el resumen en Kabatek (1997: 238f.) y Kabatek/Pusch (22011: 189). En la acepción de Bourdieu, la "hypercorrection" no produce infracciones del sistema sino de norma/uso/estilo. 
un acto singular que no tuviese nada que ver con la realidad lingüística de todos los días - ¿cómo se podría explicar entonces que la forma clásica (¿no existente en la comunicación real?) premaneciese a disposición para ser contaminada por una forma vulgar y convertirse, a fin de cuentas, en forma vulgar? La única solución que vemos para tal problema es la de partir del principio de que la norma clásica del latín -cual que fuera su vitalidad ${ }^{31}$ - estaba presente también en la vida de todos los días y que así había co-existencia de latín clásico y romance, lo que permitía la influencia recíproca, cf. Depreux (2002/3: 747, letra negrita F.J.):

Le charme d'un manuscrit de Trèves du $\mathrm{X}^{\mathrm{e}}$ siècle pour soigner une maladie des chevaux illustre de manière particulièrement claire l'osmose qui pouvait régner entre culture érudite et culture populaire dans les monastères : Incantatio contra equorum egritudinem quam nos dicimus spurihalz; le titre est en latin, mais le nom du mal est indiqué en langue germanique. L'étude des charmes faisant appel à diverses divinités montre que la christianisation en profondeur des monastères n'était pas accomplie, car elle supposait une acculturation de chaque génération de novices. La transcription de ces formules au gré du hasard alors qu'elles étaient officiellement considérées comme superflues, voire néfastes, illustre la continuité des traditions orales et populaires au sein de l'élite culturelle de la société du Haut Moyen Âge.

De la misma maniera se expresó Elias (1997/1939: 239):

"Auch hier [also bezüglich des Sprachverhaltens], wie bei den Umgangsformen, gibt es eine Art von Doppelbewegung: Verhöflichung bürgerlicher Menschen, Verbürgerlichung höfischer Menschen. Oder um es genauer zu sagen: bürgerliche werden durch das Verhalten höfischer Menschen, höfische durch das Verhalten bürgerlicher Menschen beeinflußt."

[Traducción F.J.: También en este caso [el del comportamiento lingüístico] acierta lo que hemos constatado en cuanto a los actos de cortesía. Quiere decir que también en el caso del comportamiento lingüístico hay una especie de movimiento doble: los borgéses son influenciados por los nobles y, viceversa, los nobles por los borgéses.]

El hecho de que haya coincidencia entre Depreux y Elias, a pesar de hablar de épocas distintas, ha contribuido a hacernos concebir al comportamiento lingüístico como un rasgo universal del comportamiento humano y como factor decisivo de la gestación del fenómeno tratado en el presente artículo.

\section{2 ¿Quién utilizaba "antes" CUM ME ("sic dicebant") y qué quiere decir "antes"?}

El tipo “CUM ME/con mí" es una innovación del latín vulgar que por haberse convertido en forma pan-rómanica (cf. Smith 2011: 270-279) sería utilizada por gran nú-

31 Cf. los escenarios descritos arriba. 
mero de los locutores del iberorromance, cf. García Turza (2004: 113, véase citación arriba, párrafo 1.). Esta constelación nos lleva a destacar un detalle muy interesante para facilitar la comprehensión del proceso: No será fácil hablar de un "ahora" y un "antes" en cuanto a la dicotomía "CUM ME versus MECUM" presentada en la glosa. Tendremos que plantear el problema de otra manera: Claro está que -hablando de una innovación (con mí respecto a la forma presumiblemente más antigua mego)- cabe presuponer un proceso de sucesión de las formas: "primero" existía mego y "luego" con mi lo que representa el orden inverso respecto del indicado en la glosa: "cum me: sic dicebant, et cum te; quod nunc elegantjus dicimus mecum, tecum". Sin embargo, para nosotros, quienes estamos indagando en el origen de la forma pleonástica "conmigo", es más importante otro aspecto: Independentemente de un "antes" y un "después" en lo referente al uso de formas distintas será crucial considerar el hecho de que "CUM ME (con mí)" y "MECUM (mego)" co-existieran, si no, no habría podido ocurrir la contaminación que nos interesa: con + mego. Resumamos aquí los distintos pasos:

\section{Según el Codex emilianense Em 31, la sucesión era:}

CUM ME $\rightarrow$
("sic dicebant")

\section{Sin embargo, teniendo en cuenta la diacronía del latín, el desarrollo sería más bien (teóricamente):}

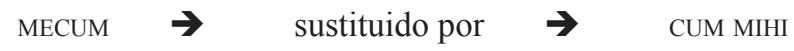

A este esquema cabe añadir las evoluciones fonéticas "naturales" así que este modelo teórico tendrá que ser adaptado como sigue:
* $\mathrm{MEGO}^{32}$
sustituido por
con mí

La verdad es que a este respecto se nos presenta una situación mucho más complicada. Cabrá, consecuentemente, ofrecer varios escenarios de posibles desarrollos de la forma en cuestión para poder escoger el que más probabilidad tenga.

\section{Variante a.) El planteamiento de Rini $(1990 a+b)$ :}

Según Rini (1990b: 61-62) no hay co-existencia de *MEGO y con mi ya que * MEGO se había convertido en forma opaca del que se desprende que no podía existir ni co-existir como forma independiente. Así que se nos presentaría el esquema siguiente:

$$
*_{\mathrm{MEGO}} \rightarrow \text { por ser opaco reforzado por } \mathrm{CON} \rightarrow \mathrm{CONMEGO}^{33}
$$

32 Aunque no está atestada esta forma (*mego) debió de existir, cf. Rini (1990a: 508): “[...] early, i.e., in preliterary times", véase también 2.3, abajo, los ejemplos (2) y (3).

33 La forma conmego está atestada en Lope de Vega, véase http://artelope.uv.es/biblioteca/textosAL/AL0566_PedroCarbonero (sub http://www.corpusdelespanol.org/web-dial/, s.v. "conmego", año: 1620, obra: Pedro Carbonero, texto utilizado para esta edición digital: Pedro Carbonero. Alicante, Biblioteca Virtual Miguel de Cervantes, 2002. Edición digital a partir de: Parte catorce de las comedias de Lope de Vega Carpio. Madrid, Juan de la Cuesta, 1620 [en Base de Datos Teatro Español del Siglo de Oro (TESO)].) Véase también: CORDE-Corpus, 


$$
\begin{array}{ll} 
& \rightarrow \text { única influencia de con mí: a nivel fonético, } \\
\text { por analogía } & \rightarrow \text { CONMEGO } \quad \mathbf{X} \text { con } m \boldsymbol{i}=\text { conmigo }^{34}
\end{array}
$$

Sin embargo, es problemático presuponer que * ${ }_{\mathrm{MEGO}}$, por ser forma opaca, ya no se empleaba, pues la glosa tratada en el presente artículo nos proporciona la información de que dicha forma era usual por lo menos en el ámbito eclesiástico. Tendríamos que recordar que no sería el primer caso de que tal cultismo influenciase otros ámbitos lingüísticos (cf. el resumen en Jodl 2018, aceptado); por ello, la posición que asume Rini parece cuestionable.

\section{Variante b.) Planteamiento alternativo al de Rini (1990a +b):}

El planteamiento alternativo que propongo aquí hace hincapié en la posibilidad de que las dos formas en cuestión no tan sólo co-existían sino que, además, fueron sujetas a un proceso de contaminación. Sin la premisa de la co-existencia, no habría sido posible que una de ellas (con $\mathrm{mi}$ ) integrara o socorriera la otra a nivel semántico. ${ }^{35}$ Ateniéndonos a este planteamiento, llegaríamos al esquema siguiente ( $\mathbf{X}$ significa "contaminado por"):

$\begin{aligned} * \text { MEGO } & \rightarrow \text { co-existencia/contaminación con con mí: } \\ & \rightarrow \text { conmego/conmigo }\end{aligned}$

Como modelo simplificado obtendríamos:

$*_{\mathrm{MEGO}} \mathbf{X} \quad$ con mi $=$ conmego/conmigo

Dado el hecho de que la variante fonética conmego -por analogía ${ }^{36}$ con con míse convirtiera en la forma definitiva conmigo tendremos que reconocer que la dimensión de la contaminación era de un tamaño impresionante o más exactamente: múltiple. Es decir que la influencia, o sea, la contaminación, no se restringió al nivel morfológico creando una simple combinación de "con" y "mego". Cuanto duró este estado de co-existencia, no será posible averiguarlo con exactitud. Lo que sí podemos constatar en el caso del tipo ${ }^{37}$ "conmigo" es que la atestación más antigua de la innovación, es decir del tipo pleonástico "cum mecum, cum tecum, cum secum", se remonta a la segunda mitad del Siglo X: Se trata de una de las Glosas Silenses (letra gordita F.J.)::38 Secum retinere uoluerit [consico kisieret tenere].

Lamentablemente, no es posible averiguar la primera aparición de formas del tipo "con mí" ${ }^{39}$ Sin embargo, teniendo en cuenta su carácter panrománico y la afirmación

s.v. "conmego": Góngora y Argote, Luis de (1615, Letrilla [Letrillas]), online : http://corpus.rae.es/cgi-bin/ crpsrvEx.dll y también Vélez de Guevara, Juan (1672, Entremés de autora de comedias), online : http://corpus. rae.es/cgi-bin/crpsrvEx.dll.

34 El cambio fonético /e/ > /i/ se debe a la analogía con mí, cf. Pascual López (2016: 27), según Rini (1990a: 508), más especifícamente, a la analogía con con mí.

35 Es aquí donde también entra en el juego la cuestión de la "junk morfológico", cf. Lass (1990). Nos dedicaremos a este problema más adelante.

36 Véase arriba, nota 34.

37 Buscando tokens, se han tomado en cuenta variantes gráficas/fonéticas como conmego, con mego, con migo, commigo, commego.

38 Véase: http://habilis.udg.edu/ info/webs/TACT/TNLASXII.TXT [26.12.2018] y también Urrutia Cárdenas/ Alvarez Alvarez (2001: 156).

39 Véase Smith (1999), Smith (2005), Smith (2006), Smith (2011: 270-280). Cf. también Rohlfs (1968: 137, 
metalingüística de "sic dicebant" (véase arriba, 1. y 2.2), se podrá constatar -con bastante probabilidad- que el tipo "con mí" es anterior a la innovación (tardía) "conmigo / conmego etc.". Siendo la forma "MECUM (mego)" una forma del latín clásico, será la variante más vieja, que existía "desde siempre". Consecuentemente, la época de co-existencia de "mego" y "con mí" tendría que ser datada al período "entre antigüedad tardía y aprox. 950-999" (950-999 como término "ante quem").

\section{3 ¿Quién utiliza "ahora" MECUM ("nunc [...] dicimus) y qué quiere decir "ahora"?}

La glosa insinúa que hay un "antes" que se corresponde al uso "incorrecto/no elegante" (CUM ME) y un "ahora" que - a su vez- se corresponde al uso "elegante"/correcto (MECUM). Se insinúa también ("elegantjus") que MECum se prefería en grupos sociales cultos. Sin embargo, la dialectología románica nos enseña que el uso del tipo MECuM no era exclusivo de los locutores cultos: El tipo MECUM (forma y función clásicas) se encuentra en algunas variedades diatópicas italianas así que su uso no se limitaría al latín clásico, sino que sería común en el latín vulgar también, cf. Rohlfs (1968: 139s., $\S 443)$. Además, en variedades diatópicas del iberorromance también se hallan restos del tipo MECUM, sin embargo con función cambiada (MECUM ha asumido la función de sujeto), cf. Cano González (2009/1981: 126) y: www.corpusdelespanol.com:

(2) Pudo ser que él fuesse de antes de cristal, de oro y zafiros, mas aora dezir puedo que es ya como tigo y migo. ${ }^{40}$

(3) Vemos que los traen señoras de todas clases, y que muchas de ellas frecuentan los sacramentos, confesándose con hombres sabios que las absuelven y se lo permiten; conque no debe de haber en eso tanto mal como a usted se le figura. - Dobremos la hoja, sobrino - respondió el familiar -; que quizá nos meteremos en cosas muy hondas, donde ni tigo ni migo podamos salir. ${ }^{41}$

Como consecuencia de un proceso de reanálisis, las formas migo / tigo ya no funcionaban -en las variedades íberorromances en cuestión- como formas de objeto preposicional, sino más bien -como decíamos- como sujeto, cf. Cano González (2009/1981: 126). Las formas vernáculas mencionadas que continúan el tipo clásico MECUM existen también en variedades italianas pero -contrariamente a las formas iberromances que acabamos de citar (uso pleonástico o uso como pronombre de sujeto)-42 no sólo con estos usos "erróneos" o "vulgares", sino también con la función original conservada, cf. respecto del dialecto de Parma Rohlfs (1968: 139s., §443):

(4) “[...] parmigiano mek, tek, nosk, per esempio gní nosk 'venite con noi' [...].”43

$\S 442)$, donde se remite a fuentes medievales.

40 Fecha: 1599, Fuente: Título Romancero spiritual, Autor: Valdivielso, José de (1560-1638), véase: http://www. cervantesvirtual.com/FichaObra.html?Ref=715 [26.12.2018].

41 Fecha 1742, Título Fray Gerundio de Campazas Autor Isla, José Francisco de (1703-1781) Fuente: http://www. cervantesvirtual.com/FichaObra.html?Ref=130 [26.12.2018].

42 Los usos "erróneos" o "vulgares" mencionados con referencia al íberorromance se encuentran también en el ítalorromance, cf. Rohlfs (1968: 139, §443).

43 gní nosk "venite con noi' < lat. VeNITE NO(BIS)CUM. 
Este párrafo ya deja translucir que la presunta alternativa de un "ahora" y un "antes" probablemente no existía como tal. Nos encontraremos, al contrario, ante una situación que se podrá describir cómo situación de co-existencia de dos formas gramaticales en vez de una sucesión del tipo "ahora" vs. "antes". Este planteamiento lo vamos a profundizar en el párrafo siguiente.

\section{4 ¿Cómo podemos interpretar "elegantjus” (“nunc elegantjus dicimus”)?}

Como dijimos arriba, la glosa insinúa un proceso de corrección que implica que hay un "antes" (uso común/incorrecto) y un "ahora" (uso más elegante). Dijimos también que esta idea de sucesión de formas gramaticales tendrá que ser reemplazada por la idea de co-existencia de las formas. A esto hay que añadir que "elegantjus" es un testimonio precoz de la conciencia lingüística medieval. Además: Aunque MECUM fuera "elegantjus", no se impuso. La forma que sí se impuso "aún" no figura en la glosa (del siglo X). Cabe destacar a este respecto que la manera de la que se expresa el autor de la glosa podría dar la impresión de que éste no era consciente del hecho de que la forma que considera como la actual y que presenta como innovación fuera la más antigua en vez de la innovadora. Pero esto no es muy probable ya que el autor representa el uso de la forma en cuestión (MECUM) como "elegantjus". Además, la forma MECUM pudo ser considerada como "más elegante" tan sólo por alguien que sabía que en el latín CLÁSICO era la forma correcta.

\section{5 ¿Cómo se puede explicar que no se impusieran ni la forma previsible cum ME, ni el-como parece-igualmente previsible MEcuM?}

Tomemos como punto de partida el hecho de que en el ámbito del subestándar latino se gestó la tripartición/co-existencia de las formas siguientes:

Tripartición (en el latín vulgar/protorromance):

a.) MECUM clásico, forma y uso clásicos:*Vas mego.

$\rightarrow$ en vía de extinción, hoy: extinto en iberromance, presente en algunos dialectos italianos

2.) MECuM reanalizado como sujeto: [él] es como [...] mego.

$\rightarrow$ sobrevivió, pero está relegado a variedades subestándar del español

3.) Innovación panrómanica CUM ME: Vas con mí.

$\rightarrow$ sobrevivió, pero está relegado a variedades subestándar del español

Así que en ámbitos de lenguaje inculto, como formas, seguían co-existiendo: mecum (mego) y cum me (con mí). Como dijimos, no se impuso ni la una ni la otra sino el resultado de la contaminación de las dos:

$$
\begin{array}{ll}
\text { MECUM X } & \text { CUM ME }=\text { CUM MECUM } \\
\text { mego } \mathbf{X} & \text { con mi }=\text { conmego/conmigo }
\end{array}
$$

Si nos preguntamos cómo pudo ocurrir esta contaminación, se nos ofrece -otra 
vez- un planteamiento sociolingüístico que sería el siguiente: La glosa nos demuestra que la conciencia de lo que era "más elegante" seguía más o menos intacta. Cundía, sin embargo, el uso de la forma alternativa "CUM ME" que al gestarse el primer estándar romance a pesar de todo no pudo imponerse: Los hablantes preferirían una variante más prestigiosa cuyas reglas de empleo y formación -mientras tanto-ignoraban. Con otras palabras: En lo referente al uso de la forma MECUM/mego cundiría una idea imprecisa/vaga de cómo utilizar la forma o más exactamente: Se podía haber quedado una especie de "junk”/“basura" (cf. Lass 1990, Espinosa Elorza 2008 y Jodl 2018, aceptado). Lass (ibd.) esgrime la opinión de que "junk” morfológica puede desatar procesos de cambio lingüístico. Este fenómeno, que designa con "exaptation" $"$, puede ser definido como sigue, cf. Lass (1990: 80):

Exaptation [...] is the opportunistic ${ }^{45}$ co-optation of a feature whose origin is unrelated or only marginally related to its later use. In other words (loosely) a 'conceptual novelty' or 'invention'.

La designación "exaptation" se remonta a un concepto acuñado por la biología evolucional, cf. Gould/Vrba (1982), Lass (1990: 80) y el resumen de Traugott (2004: 133). Más tarde, Gould restringe el concepto de la "exaptation" como sigue, cf. Gould (1983: 171): ${ }^{46}$

We wish to restrict the term adaptation only to those structures that evolved for their current utility; those useful structures that arose for other reasons, or for no conventional reasons at all, and were fortuitously available for other changes, we call exaptations.

Este concepto se puede transferir a la gestación de la forma pleonástica CONMECUM afirmando que la motivación para utilizar, a pesar de todo, esta forma incorrecta (el hipercorrectismo) sería su prestigio, cf. un caso análogo descrito por Brunot/ Bruneau (1933: 557s.):

Il arrive que le subjonctif prenne, dans un certain nombre de phrases, un aspect littéraire et élégant, tandis que l'indicatif est banal et populaire. Certains écrivains plus scrupuleux que corrects commencent à employer le subjonctif à contre-sens: des subjonctifs de 〈snobisme〉 apparaissent un peu partout.

Es muy fácil constatar que la analogía consiste en los hechos siguientes: Se ignora la regla de utilización exacta. Sin embargo, se emplea la forma porque los locutores a la hora de emplearla de manera incorrecta, no son conscientes de la infracción de la norma o más bien: Están convencidos de que se trata de la forma correcta. Otro ejemplo:

44 Cf. las precisiones conceptuales, concretamente "capitalization / ad-functionalisation" en Pountain (2000) y "refunctionalization" en Smith (2011). El último concepto ("refunctionalization") se corresponde al concepto de "capitalization / ad-functionalisation" acuñado por Poutain, ibd.

45 Vgl. Traugott (2004: 136): "Change is not deterministic or teleological".

46 En su resumen, Traugott (2004: 134) se refiere a los siguientes ejemplos "clásicos": "In biology standard examples are the redeployment of reptiles' feathers that served thermoregulatory purposes for flight, or the redeployment of vertebrates' respiratory and digestive devices for sound production.". 
(5) *das Hause en vez de das Haus, cf. Jodl (2006: 12-16 y Jodl 2018: 275-280; con casos análogos);

En alemán, el nominativo de los sustantivos (como “das Haus") no se puede marcar por desinencia. Sin embargo, através de Google se puede detectar un número elevado de ocurrencias de "das Haus $\underline{\boldsymbol{e}}$ ". No quiere decir que no existan substantivos con la desinencia -e. Pero se tiene que tener en cuenta que, si esta desinencia está justificada, se trata de otro caso flexional, el dativo, y además: Esta desinencia del dativo se ha vuelto obsoleta, ha sobrevivido tan sólo en expresiones fijas (a las que el arcaísmo de la desinencia confiere más prestigio) como p. ej.:

$\underline{\text { In }}$ diese $\underline{\boldsymbol{m}}$ Falle que coexiste como variante más prestigiosa de:

In diesem Fall_ (es la forma actual "normal", sin carecer de prestigio)

Se podría decir que el dativo brinda, como una especie de "junk" (véase arriba), la posibilidad morfológica de aumentar el prestigio añadiendo la "vieja" desinencia del dativo. Los locutores se sirven de esta posibilidad "legítima", o sea, la de añadir la vieja desinencia, sobre todo en el contexto siguiente:

$\rightarrow$ denominaciones de dinastías EN LA FORMA DEL DATIVO LOCATIVO

$$
\begin{array}{ll}
\text { p. ej.: ¿Dónde? } & \rightarrow \text { im Hause Windsor } \\
& \rightarrow \text { im Hause Borbón/Bourbon } \\
& \rightarrow \text { im Hause Wittelsbach }
\end{array}
$$

En estos casos, la conservación de la vieja - e del dativo no se considera como exagerada dado el contexto "de prestigio". Lo interesante es: El ensanchamiento ilegítimo del uso de la -e hacia los nominativos ${ }^{47}$ se observa siempre en los mismos contextos:

\section{$\rightarrow$ prestigio / nobleza \\ $\rightarrow$ acto consciente de los locutores}

Otros ejemplos:

(6) “secundum meum savirum”, cf. Cerquiglini (2007: 123):

"Parmi les formules relevées par F. Ewald [1964] notons cette étonnante secundum meum savirum, dont le savirum n'est que le grossier habillage latin d'un mot roman"

(7) “ $[\mathrm{cum}]$ animabus” en vez de $[\mathrm{cum}]$ animis $^{48}$

Aunque los ejemplos (6) y (7) corresponden sólo parcialmente al caso de "conmigo" -véase la clasificación de los ejemplos que estableceré en las conclusiones-cabe

\footnotetext{
$47 \quad$ No se trata de la utilización de una alternativa existente justificada, como en el caso del dativo, sino de la creación de una nueva forma o una nueva regla.

48 Fuente: Doc 2 (804) de los Cartularios de Valpuesta, cf. Ramos Remedios (2000: 67).
} 
destacar que los ejemplos, todos juntos, contribuirán a explicar el fenómeno de la gestación de la forma pleonástica en cuestión. ${ }^{49}$

\section{3. ¿Qué papel desempeñan en nuestro caso planteamientos como la morfología “junk", la "exaptación”, la "capitalización” y "la refuncionalización”?}

Como hemos visto en el párrafo 2.5, el concepto de la "junk morphology", o sea, la "exaptation" (Lass 1990, Espinosa Elorza 2008), entra en el marco del fenómeno que estamos tratando aquí. Sin embargo, cabe involucrar a este tema la pregunta de qué papel desempeñan los conceptos de la "capitalization" (Pountain 2000) y de la "refunctionalization" (Smith 2011) en cuanto al ejemplo de MECUM X CUM ME = CUM MECUM, es decir, mego $\mathbf{X}$ con mi = conmego/conmigo.

A propósito de la capitalización se trataría, concretamente, de hacer la pregunta de si en nuestro caso un elemento lingüístico existente y estable adquiere un nuevo valor. ¿Pero cuál sería?

A propósito de la refuncionalización, a su vez, tendríamos que preguntarnos si en cuanto a nuestro caso un elemento que amenaza con caer en desuso, en otras palabras: casi inexistente, se usa con un nuevo valor. ¿Pero cuál sería?

Visto que en nuestro caso no se produce en absoluto la adquisición de nuevos valores, no acierta ni el concepto de la capitalización, ni el de la refuncionalización.

Tenemos, sin embargo, que precisar lo dicho: Por (nuevo) valor se entiende "función gramatical, morfosintáctica, con implicaciones semánticas". Es éste el elemento que no acierta por lo que toca a nuestro ejemplo. Puede haber un nuevo valor en la nueva forma "CUM MECUM/conmigo", pero este valor tiene una dimensión exclusivamente sociológica ("prestigio") en vez de una función semántico-gramatical.

El punto donde el caso de "con mi X MECUM = CUM MECUM" toca el concepto de la morfología "junk"/"exaptation" se halla en el criterio de "un recuerdo difuso de una forma obsoleta genera una innovación lingüística”, sin que se cree un nuevo valor semántico-gramatical. Lo que se crea es un nuevo valor social performado por una forma lingüística. Es éste el rasgo distintivo de nuestro caso concreto - rasgo que no permite clasificarlo en una de las teorías descritas arriba.

49 Otro ejemplo de este tipo sería el caso siguiente, cf. http://www.esmaelmorais.com.br/2016/11/mesoclise-dodia-impichar-me-ao/ [26.12.2018]; aporte en el forum de parte de Edison Carvalho 25 de novembro de 2016 em 20:29: (8) "E que além do depoimento lavrado na PF, apareca também a FITA....................!!!!!! Deixemos o Trombolho (se preferirem: Deixe-mo-o)..................sic, NU..................!!!!" [letra negrita: F.J.]. El problema es: La forma empleada en la citación (Deixe-mo-o) no existe en absoluto. La intención del locutor sería expresar el concepto de: Deixemo-lo (Dejémoslo en español). Lo confundiría con una forma obsoleta en el portugués brasileño, o sea, la mesoclisis -todavía usual en el portugués europeo al construir formas del futuro y del potencial- como en p. ej. Dar-te-ei/Chamá-lo-ei = esp. antiguo Dar te hé/Llamar lo hé = esp. mod. : Te daré/Lo llamaré, , cf. Jodl (2016b, espec. 170-174). Quisiera agradecerle a Claudio Cardinali/ Universität Köln por haberme confirmado la plausibilidad de la interpretación que acabo de proponer. Ésta se basa en la presuposición de que la elección de la forma "deixe-mo-o" no está motivada por plena ironía. En Jodl (2013: 848s.) describí un caso análogo, concretamente la confusión de los paradigmas portugueses "subjuntivo do futuro" vs. "infinitivo pessoal" que se produce en una variedad portuguesa hablada en el norte del Uruguay) y influenciada por el español: El "Fronterizo". 
Así habrá que establecer un nuevo planteamiento, el de la morfología de prestigio que abarque los criterios del saber lingüístico y de la koineización.

\section{Propuesta de un planteamiento ulterior: la morfología de prestigio y sus implicaciones con el saber y el comportamiento lingüísticos y la koineización}

Según lo dicho en el párrafo precedente, me decanto por colocar nuestro fenómeno dentro de una categoría aparte que nombré "morfología de prestigio". Este concepto se basa en los siguientes criterios decisivos:

a.) El morfema en cuestión fue elegido conscientemente

b.) La motivación para la formación es: aumentar el prestigio de la forma y del hablante

c.) El patrón no se encuentra en las formas, sino que en el comportamiento (lingüístico) de los hablantes: Se aspira a utilizar formas más prestigiosas.

Dado que los criterios a.) y b.) ya han sido tratados en los párrafos de arriba, me centraré ahora en el criterio c.), o sea, en el comportamiento (lingüistico) de los hablantes.

Estos fenómenos están estrechamente vinculados con la morfología de prestigio. Quiere decir que los hablantes eligen deliberadamente ciertas formas lingüísticas elección que depende del saber lingüístico. Éste, sin embargo, no es constante, es normal que se produzcan pérdidas de este saber específico que conllevan la formación de nuevas formas y reglas que -independentemente de su corrección- se basan en su presunto (o verdadero) prestigio. Pero la elección individual de una forma de prestigio (sea "natural" o acuñada) aún no explica el cambio lingüístico, es decir, la generalización. Es aquí donde entra en juego el fenómeno de la koineización - fenómeno que tenemos que tomar en cuenta ya que la datación del Codex 31 entra en el marco del proceso de koineización que recorrió el castellano antiguo (Tuten 2003). Tratar el fenómeno de la koineización significa que tenemos que hacernos la pregunta de qué ventaja comunicativa podía tener la forma redoblada conmego/conmigo para que se impusiera y además de si tenía una transparencia elevada y si seguía un patrón más frecuente.

Si comparamos la imposición de conmego/conmigo con p. ej. la imposición de la no-amalgamación de los elementos "PREPOSICIÓN + artículo" en el caso de IN + ILLU (que produjo la forma analítica en el en vez de la amalgamada "no" (cf. Tuten 2003: 114s.) tendremos que concluir que es dificil encontrar tales ventajas en cuanto al caso de "conmego/conmigo versus con mi": La única motivación para preferir la forma pleonástica sería el prestigio. Con el criterio del prestigio está vinculado otro criterio que explica el éxito positivo del fenómeno dentro del marco de la koineización: El feature sería utilizado por un peer group, cf. Labov (1963 y 2010) y lo expuesto arriba.

\section{Conclusiones}

Podemos dar el siguiente resumen de lo expuesto acerca de la gestación de la forma tautológica de CUM MECUM > conmigo: 
a.) Coexistían en el proto-iberorromance las variantes
Tipo 1: *Vas mego
Tipo 2: *Vas con mí.

b.) En la variedad estándar, la contaminación de los dos se gestaría del modo siguiente:

A través del contacto con el ámbito culto en el que se había mantenido el uso correcto de la forma MECUM, pudo mantenerse en el ámbito menos culto una idea vaga ("junk" morfólogica) de la expresión MECUM sin que los locutores en cuestión supiesen emplearla correctamente. Consecuentemente, los locutores menos cultos utilizaban MECUM de manera reanalizada, o sea, reforzada por CUM. La forma correcta MECUM, que no se impondrá, transfiere su prestigio a la forma vencedora CUMMECUM. Es el criterio del prestigio que desempeña el papel de factor decisivo para su imposición.

c.) Puede haber contribuido la situación de la koineización (Tuten 2003) dado que en el caso de CUM MECUM $>$ conmigo se trataría de un peer-group-feature (...nunc elegantjus dicimus..., véanse arriba, 1. y 4.)

Para caracterizar mi concepto de morfología de prestigio propondría el resumen siguiente: El concepto de morfología de prestigio se puede considerar como la extensión del principio de la "exaptación". Esta extensión consiste en el énfasis del auto-concepto del hablante - auto-concepto que puede desempeñar el papel de motor de innovaciones lingüísticas en virtud de la aspiración del hablante a aumentar su prestigio utilizando formas de prestigio. Concretamente, se pueden distinguir los procesos siguientes:

Escenario a): Se añaden desinencias que, en una primera fase, no corresponden al sistema vigente (como en el caso de "*das Hause") pero que pueden, en un segundo paso, cambiar el sistema convirtiéndose en nuevos morfemas integrantes del sistema. El proceso del "añadir" puede también ser considerado como la sustitución de un morfema cero por un morfema explícito.

Escenario b) Se reemplazan, sin necesidad semántica, morfemas ya integrantes de un sistema lingüístico por nuevos elementos lingüísticos considerados como más prestigiosos, como en el caso de "cum mihi / cum mecum".

\section{Bibliografía}

\section{Fuentes primarias:}

Alcuinus (735 - 804), "Vita Beatissimi Richarii Presbyteri”, en: Migne Jacques-Paul, Patrologia latina 101, 681-694; véase también: Vie de Saint Riquier (Alcuin 801), online: http://www.documentacatholicaomnia.eu/04z/z_0735-0804_Alcuinus_Vita_Beatissimi_ Richarii_Presbyteri_MLT.pdf.html [21.11.2018]

De Callières, François (1693/1972): Des Mots à la mode - Du bon et mauvais usage dans les manières de s'exprimer, Genève: Slatkine Reprints.

Gregorio de Tours: Krusch, Bruno/Levison, Wilhelm (1937, eds.): Scriptores rerum Merovingicarum 1,1: Gregorii Turonensis Opera. Teil 1: Libri historiarum X. Hannover 1937 (Monumenta Germaniae Historica, Digitalisat) [= edición estándar de las Historiae] 
Hincmar de Reims: Capitularia regum Francorum, t. 2, éd. Alfred Boretius et Victor Krause, Hannover, Monumenta Germaniae Historica, 1890, no 297, p. 436 (Epistola synodi Carisiacensis ad Hludowicum regem Germaniae directa, c. 12).

Ekkehard IV, en: MGH, Abt. I: Scriptores 2 [Scriptores rerum Sangallensium. Annales, chronica et historiae aevi Carolini], $121 \mathrm{~s}$.

= Ekkehard IV: Casus Sancti Galli. Editionis textum paravit Hans F. Haefele. Ekkehard IV. Sankt Galler Klostergeschichten. Übersetzt von Hans F. Haefele (Ausgesuchte Quellen zur deutschen Geschichte des Mittelalters. Freiherr-vom-Stein-Gedächtnisausgabe X), Darmstadt: Wissenschaftliche Buchgesellschaft.

Notker Balbulus, en: Gesta Karoli magni imperatoris, éd. Hans F. Haefele, Berlin, Monumenta Germaniae Historica, 1959, p. 4-5 (I, 3).

Il Novellino. Das Buch der hundert alten Novellen, italienisch-deutsch, ed./trad. Janós Riesz (Universal-Bibliothek, vol. 8511), Stuttgart, Reclam, 1988.

Vie de Saint Éloi = Vita Eligii (prol. MGH SS rer. merov. IV, 634-761, espec. 664).

\section{Literatura secundaria:}

Berschin, Walter (1986): Biographie und Epochenstil im lateinischen Mittelalter. Vol. 1: Von der Passio Perpetuae zu den Dialogi Gregors des Großen, Stuttgart: Hiersemann.

Beumann, Helmut (1964): “Gregor von Tours und der Sermo rusticus”, en: Repgen, Konrad/ Skalweit, Stephan (eds.): Spiegel der Geschichte: Festgabe für Max Braubach zum 10. April 1964, 69-98.

Bonnet, Max (1890): Le latin de Grégoire de Tours, Paris: Hachette.

Bork, Hans Dieter (2006): "Sprachkontakte: Latein und Galloromanisch", en: Ernst, Gerhard et al. (edd.), 1582-1590.

Bourdieu, Pierre (1984/2002): "Le marché linguistique”, en: Questions de sociologie. Pierre Bourdieu (ed.), Paris: les Éditions de Minuit, 121-138.

Bourgain, Pascale/Heinzelmann, Martin (1997): “L'œuvre de Grégoire de Tours: la diffusion des manuscrits", en: Nancy Gauthier y Henri Galinié (eds.): Grégoire de Tours et l'espace gaulois. Actes du congrès international, Tours, 3-5 novembre 1994, Tours: Association Grégoire 94, 273-317.

Brunot, Ferdinand / Bruneau, Charles (1933): Précis de grammaire historique de la langue française, Paris: Mousson.

Buchner, Rudolf (1942, recensión): Krusch, Bruno/Levison, Wilhelm (1937, eds.): Scriptores rerum Merovingicarum 1,1: Gregorii Turonensis Opera. Teil 1: Libri historiarum X. Hannover 1937 (Monumenta Germaniae Historica), en: ZRG, gA, 62, 404-414.

Buchner, Rudolf (1951, recensión): Krusch, Bruno/Levison, Wilhelm (1937, eds.), véase arriba, en: $Z R G, g A, 68,465-470$.

Buchner, Rudolf (1955, recensión): Krusch, Bruno/Levison, Wilhelm (1937, eds.), véase arriba, en: $Z R G, g A, 72,277-278$.

Buchner, Rudolf ( ${ }^{9} 2000$, ed.): Gregor von Tours, Zehn Bücher Geschichten, Zweiter Band, Buch 1-5 (Ausgewählte Quellen zur deutschen Geschichte des Mittelalters / Freiherr vom Stein-Gedächtnisausgabe 3), Darmstadt: Wissenschaftliche Buchgesellschaft.

Cano González, Ana María (2009): El habla de Somiedo, Oviedo: Academia de la Llingua Asturiana = (1981): El habla de Somiedo (Occidente de Asturias), Santiago: Universidad de Santiago.

Cerquiglini, Bernard (72007): La naissance du français, (1a edición 1991), Paris: PUF.

CORDE = Corpus diacrónico del Español, Real Academia de la Lengua Española, http://corpus.rae.es/cordenet.html 
Coseriu, Eugenio (1974/1958): Synchronie, Diachronie und Geschichte. Das Problem des Sprachwandels. München: Fink. (Versión original: Syncronía, diacronía e historia. El problema del cambio lingüistico. Montevideo 1958).

Delhees, Karl H. (1994): Soziale Kommunikation: Psychologische Grundlagen für das Miteinander in der modernen Gesellschaft, Opladen: Westdeutscher Verlag.

Depreux, Philippe (2002/3): “Ambitions et limites des réformes culturelles à l'époque carolingienne", en: Revue historique 623, 721-753.

online: https://www.cairn.info/revue-historique-2002-3-page-721.htm [21.11.2018]

Elias, Norbert (1997/1939): Über den Prozeß der Zivilisation: soziogenetische und psychogenetische Untersuchungen, Band 1: Wandlungen des Verhaltens in den weltlichen Oberschichten des Abendlandes, Frankfurt/Main: Suhrkamp.

Espinosa Elorza, Rosa María (2008): “La semántica en los procesos de cambio categorial: las palabras gramaticales en un diccionario histórico”, en: Diccionario histórico: nuevas perspectivas lingüisticas, María Pilar Garcés Gómez (ed.), Madrid y Frankfurt am Main: Iberoamericana Verfuert.

Ernst, Gerhard et al. (2006, edd.): Romanische Sprachgeschichte / Histoire linguistique de la Romania. Ein internationales Handbuch zur Geschichte der romanischen Sprachen, 2. Teilband, Berlin/New York: de Gruyter.

Ewald, Konrad (1964): "Formelhafte Wendungen in den Straßburger Eiden", en: Vox Romanica 23 , S. 35-55.

Ferguson, Charles F. (1959): "Diglossia”, en: Word. Journal of the Linguistic Circle of New York 15, 325-340.

Fuhrmann, Manfred (1994): Rom in der Spätantike. Porträt einer Epoche, München/Zurüch: Artemis \& Winkler.

García Turza, Claudio/García Turza, Javier (1995): "La datación y procedencia de las Glosas Emilianenses y Silenses: Anotaciones críticas a los nuevos planteamientos", en: Brocar 19, 49-64. Online: http://www.vallenajerilla.com/berceo/garciaturza/anotacionesglosas. htm [21.11.2018]

Goffart, Walter (1987/1989a): "From Historiae to Historia Francorum and back again. Aspects of the textual history of Gregory of Tours", en: Religion, Culture, and Society in the Early Middle Ages: Studies in Honor of Richard E. Sullivan, Studies in Medieval Culture 23, Thomas F.X. Noble y John J. Contreni (eds.), Kalamazoo: Medieval Institute Publications, 55-76.

Reimprimido 1989 en: Goffart, Walter (ed.): Rome's Fall and After, London et al.: Hambledon, 225-274.

Goffart, Walter (1989b): The narrators of barbarian history (A.D. 550 - 800) : Jordanes, Gregory of Tours, Bede, and Paul the Deacon, Princeton: Princeton University Press.

Gould, Stephen J. (1983): Hen's Teeth and Horse's Toes. Further Reflections in Natural History, New York: Norton.

Gould, Stephen J./Vbra, Elizabeth S. (1982): "Exaptation - a missing term in the science of form", en: Paleobiology 8, 4-15.

Heen, Katrien (1991): "Audire, legere, vulgo : an attempt to define public use and comprehensibility of Carolingian hagiography", en: Latin and the Romance Languages in the Early Middle Ages, Roger Wright (ed.), London: Routledge, 146-163.

Jodl, Frank (2002/3): „Die “Architektur der Sprache“ von unten gesehen: Zur Klassifikation der romanischen Idiome Norditaliens", en: Horizonte 7, Narr: Tübingen, 169-186.

Jodl, Frank (2006): "Form und Funktion: Welchen Beitrag kann die Sprachwissenschaft im Sprachunterricht leisten? Gedanken zu einem fachübergreifend angelegten Vorgehen", 
en: Zeitschrift für Fremdsprachenforschung 17/1, S. 3-33.

Jodl, Frank (2010): "Die Dichotomie Schriftlichkeit/Mündlichkeit und die frühmittelalterliche Sprachgeschichte Oberitaliens: Grenzbereiche und Übergänge”, en: Zeitschrift für Romanische Philologie 126/1, S. 134-156.

Jodl, Frank (2013): "Uruguay", en: Weltsprache Spanisch: Variation, Soziolinguistik und geographische Verbreitung des Spanischen. Handbuch für das Studium der Hispanistik, Sandra Herling y Carolin Patzelt, Stuttgart: Ibidem, 825-852.

Jodl, Frank (2016a): "Guerra del Castellano und Convivencia: Die Aktualität des Mittelalters für die akademische Lehre", en: Hispanorama 152, 94-105.

Jodl, Frank (2016b): “Corporate Identity 'Portugiesisch' - historische Sprache versus Unterrichtsfach: Das Problem der Identität des Portugiesischen im Spiegel ausländischer Lehrwerke", en: Lusorama 105/106, 161-200.

Jodl, Frank (2018, aceptado): “(Re)-Latinisierung und gesprochene Sprache: der spanische persönliche UND flektierte Infinitiv im Vergleich zu seinem portugiesischen Korrelat", en: Lusorama.

$=$ "(Re)-latinization and spoken language: the Spanish personal AND inflected infinitive". 48th Annual Meeting of the Societas Linguistica Europaea, 2nd - 5th September 2015, Leiden/NL.

Jodl, Frank (2018): Fremdsprachenunterricht und Linguistik-Studium: 'Wozu brauchen wir das eigentlich?' Eine Orientierungshilfe für sprachübergreifendes Lehren auf kontrastiver Basis, Stuttgart: Ibidem. (Romanische Sprachen und ihre Didaktik 66).

Kabatek, Johannes (1997): “Zur Typologie sprachlicher Interferenzen”, en: Neue Forschungsarbeiten zur Kontaktlinguistik, Wolfgang W. Moelleken y Peter J. Weber (eds.), Bonn: Dümmler, 232-241.

Kabatek, Johannes (2005): "Über Trampelpfade, sichtbare Hände und Sprachwandelprozesse", en: Unsichtbare Hand und Sprecherwahl. Typologie und Prozesse des Sprachwandels in der Romania, Thomas Stehl (ed.),Tübingen: Narr 155-174.

Kabatek, Johannes/Pusch, Claus D. ('2011): Spanische Sprachwissenschaft, Tübingen: Narr. Koch, Peter (1997): "Diglossie in Frankreich?", en: Frankreich an der Freien Universität. Geschichte und Aktualität. Beiträge zur Ringvorlesung ,Frankreich an der Freien Universität. Geschichte und Aktualität‘, Winfried Engler (ed.), Stuttgart: Steiner, 219-249.

Koch, Peter/Oesterreicher, Wulf (1985): "Sprache der Nähe - Sprache der Distanz. Mündlichkeit und Schriftlichkeit im Spannungsfeld zwischen Sprachtheorie und Sprachgeschichte", en: Romanistisches Jahrbuch 36, 15-43.

Koch, Peter / Oesterreicher, Wulf (2011/1990): Gesprochene Sprache in der Romania: Französisch, Italienisch, Spanisch, 1a edición 1990, Tübingen, Niemeyer. (Romanistische Arbeitshefte 31).

Kramer, Johannes (1998): Die Sprachbezeichnungen Latinus und Romanus im Lateinischen und Romanischen, Berlin: Erich Schmidt.

Labov, William (1963): “The social motivation of a sound change", en: WORD 19, 273-309.

Labov, William (2010): Principles of Language Change. Volume 3: Cognitive and cultural factors, Chichester: Wiley-Blackwell.

Lass, Roger (1990): «How to do things with junk: exaptation in language evolution», en: Journal of Linguistics 26, 79-102.

Lass, Roger (1997): Historical Linguistics and Language Change. Cambridge/UK: Cambridge University Press.

Müller, Roman (2001): Sprachbewusstsein und Sprachvariation im lateinischen Schrifttum der Antike, München: Beck. 
Pascual López, Xavier (2016): Historia de la lengua española, tema 4, El castellano medieval (hasta el siglo XV),

Online: $\quad$ https://xavierpascuallopez.jimdo.com/historia-de-la-lengua-espa\%C3\%B1ola/ [21.11.2018]

Pierno, Franco (2006): "Storia del linguaggio religioso nella Romania: italiano, sardo, Alpi orientali", en: Ernst et al. 2006, 2070-2082.

Plassmann, Alheydis (2006): Origo gentis : Identitäts- und Legitimitätsstiftung in früh- und hochmittelalterlichen Herkunftserzählungen, Berlin: Akademie-Verlag.

Pountain, Christopher J. (2000): “Capitalization”, en: Historical Linguistics 1995: selected papers from the 12th International Conference on Historical Linguistics, Manchester, August 1995, Volume 1, General Issues and non-Germanic languages, John Charles Smith y Delia Bentley (eds.), Amsterdam \& Philadelphia: John Benjamins, 295-309.

Ramos Remedios, Emilia (2000): Los cartularios de Santa María de Valpuesta. Análisis lingüístico, Donostia/San Sebastián: Eusko Ikaskuntza.

Ramos Remedios, Emiliana (2016): "El nacimiento de la conciencia lingüística castellana en la documentación anterior al siglo XII. Los textos de Valpuesta”, en: El naixement de la consciència lingüistica a l'edat mitjana, Francisco Javier Terrado Pablo y Flocel Sabaté i Curull (eds.), Lleida: Pagès Editors, 61-96.

Reutner, Ursula (2009): Sprache und Tabu: Interpretationen zu französischen und italienischen Euphemismen, Tübingen: Niemeyer.

Reutner, Ursula/Schwarze, Sabine (2011): Geschichte der italienischen Sprache. Eine Einführung, Narr: Tübingen.

Richter, Michael (1983): “À quelle époque a-t-on cessé de parler latin en Gaule ? À propos d'une question mal posée", en: Annales 38-2, 439-448.

Rini, Joel (1990a) : "On the chronology of Spanish conmigo, contigo, consigo, and the interaction of phonological, syntactic and morphological processes", en: Hispanic Review. 58. 4, 503-512.

Rini, Joel (1990b) : "Excessive analogical change as an ímpetus for lexical loss - Old Spanish connusco, convusco", en: Romanische Forschungen. CII. 1: 58-64.

Rohlfs, Gerhard (1968): Grammatica storica della lingua italiana e dei suoi dialetti, vol. 2: Morfologia, Torino: Einaudi.

Smith, John Charles (1999): "The refunctionalization of a pronominal subsystem between Latin and Romance", en: Oxford University Working Papers in Linguistics, Philology \& Phonetics 4, 141-156.

Smith, John Charles (2005): "Some refunctionalizations of the nominative-accusative opposition between Latin and Gallo-Romance", en: A Companion in Linguistics: a Festschrift for Anders Ahlqvist on the occasion of his sixtieth birthday, Bernadette Smelik et al. (eds.), Nijmegen: De Keltische Draak, 269-285.

Smith, John Charles (2006): "How to do things without junk: the refunctionalization of a pronominal subsystem between Latin and Romance", en: New Perspectives on Romance Linguistics: selected papers from the 35th Linguistic Sympsium on Romance Languages (LSRL), Austin, Texas, February 2005. Volume II: Phonetics, Phonology and Dialectology, Jean-Pierre Y. Montreuil, (ed.), Amsterdam \& Philadelphia: John Benjamins, 183-205.

Smith, John Charles (2011): "Change and Continuity in Form-Function Relations", en: The Cambridge History of the Romance Languages. I: Structures, Martin Maiden et al. (eds.), Cambridge: CUP, 268-317. Online:

http://universitypublishingonline.org/cambridge/histories/chapter.jsf?bid=CBO9780511780134\&cid=CBO9780511780134A012 [21.11.2018] 
Traugott, Elizabeth Closs (2004): "Exaptation and grammaticalization”, en: Linguistic Studies Based on Corpora, Minoji Akimoto (ed.), Tokyo: Hituzi Syobo, 133-156.

Tuten, Donald N. (2003): Koineization in medieval Spanish. Berlin et al.: Mouton de Gruyter.

Urrutia Cárdenas, Hernán/Alvarez Alvarez, Manuela (2001): Esquema de morfosintaxis histórica del español, Bilbao: Universidad de Deusto/Departamento de Publicaciones.

Wolf, Heinz-Jürgen (1991): Glosas Emilianenses, Hamburg: Buske.

Traducción al español:

Wolf, Heinz Jürgen (1996): Las Glosas Emilianenses. Versión española de Stefan Ruhstaller, Sevilla: Universidad de Sevilla.

Wolf, Heinz Jürgen (1997): "Las glosas emilianenses, otra vez", en: Revista de Filología Románica (14/1), 597-604.

Wright, Roger (1982): Late Latin and early Romance : in Spain and Carolingian France, Liverpool/UK: Cairns. 